\title{
Rogue Wave for the Benjamin Ono Equation
}

\author{
Lili Song1, Wei Chen' ${ }^{1}$ Zhenhui Xu2 ${ }^{2}$, Hanlin Chen' \\ ${ }^{1}$ School of Science, Southwest University of Science and Technology, Mianyang, China \\ ${ }^{2}$ Applied Technology College, Southwest University of Science and Technology, Mianyang, China \\ Email: songlili29@163.com, chenweimy@yeah.net, xuzhenhui19@163.com, chenhanlin@swust.edu.cn
}

Received 16 January 2015; accepted 8 February 2015; published 13 February 2015

Copyright (C) 2015 by authors and Scientific Research Publishing Inc.

This work is licensed under the Creative Commons Attribution International License (CC BY).

http://creativecommons.org/licenses/by/4.0/

c) (i) Open Access

\begin{abstract}
In the paper, the homoclinic (hateroclinic) breather limit method (HBLM) is applied to seek rogue wave solution of the Benjamin Ono equation. We find that the rational breather wave solution is just a rogue wave solution. This result shows that rogue wave can come from the extreme behavior of the breather solitary wave for $(1+1)$-dimensional nonlinear wave fields.
\end{abstract}

\section{Keywords}

Benjamin Ono Equation, Extended Homoclinic Test Method, Homoclinic (Hateroclinic) Breather Limit Method, Rogue Wave Solution

\section{Introduction}

As is well known that solitary wave solutions of nonlinear evolution equations play an important role in nonlinear science fields, especially in nonlinear physical science, since they can provide much physical information and more insight into the physical aspects of the problem and thus lead to further applications [1]. In this paper, we will consider the Benjamin Ono (BO) equation

$$
u_{t t}+\beta\left(u^{2}\right)_{x x}+\gamma u_{x x x x}=0
$$

where $\beta$ and $\gamma$ are non-zero constants. The BO equation is one of the important nonlinear model in physics [2] [3]. By means of traveling wave method, the exact solutions of the BO equation were obtained. Using the F-expansion method and the Jacobi elliptic function expansion method to the BO equation, a series of periodic wave solutions were got [4]. Based on an improved projective Riccat equation method, the traveling wave solutions of single variable were found [5]. Applying the bilinear method and extended homoclinic test approach [6]-[10], periodic solitary wave and doubly periodic solutions for the BO equation were obtained [11]. 
In recent years, rogue waves, as a special type of nonlinear waves and also known as freak waves, monster waves, killer waves, extreme waves, abnormal waves [12], have triggered much interest in various physical branches. Rouge wave is a kind of wave that seems abnormal which is first served in the deep ocean. It always has two to three times amplitude higher than its surrounding waves and generally forms in a short time for which people think that it comes from nowhere. Rouge waves have been the subject of intensive research in oceanography [13] [14], optical fibers [15]-[17], superfluids [18], Bose-Einstein condensates, financial markets and other related fields [19]-[22]. In this work, we will apply the homoclinic (hateroclinic) breather limit method (HBLM) [23], to seek rogue wave solution of the BO equation. We take the following four steps:

Step 1

By Painleve analysis, a transformation $u=T(f)$ is made for some new and unknown function $f$.

Step 2

By using the transformation in step 1, original equation can be converted into Hirota's bilinear form $G\left(D_{t}, D_{x}, f\right)=0$, where the $D$-operator [24] is defined by

$$
D_{x}^{m} D_{t}^{n} f(x, t) g(x, t)=\left(\frac{\partial}{\partial x}-\frac{\partial}{\partial x^{\prime}}\right)^{m} \times\left.\left(\frac{\partial}{\partial t}-\frac{\partial}{\partial t^{\prime}}\right)^{n} f(x, t) g\left(x^{\prime}, t^{\prime}\right)\right|_{x^{\prime}=x, t^{\prime}=t}
$$

Step 3

Solve the above equation to get homoclinic (heteroclinic) breather wave solution by using extended homoclinic test approach (EHTA) [25].

Step 4

Let the period of periodic wave go to infinite in homoclinic (heteroclinic) breather wave solution, we can Obtain a rational homoclinic (heteroclinic) wave and this wave is just a rouge wave.

\section{Rational Breather Wave (Rogue Wave)}

The BO equation,

$$
u_{t t}+\beta\left(u^{2}\right)_{x x}+\gamma u_{x x x x}=0
$$

By Painleve analysis, let

$$
u=u_{0}+\frac{6 \gamma}{\beta}(\ln f)_{x x}
$$

where $f(x, t)$ is unknown real function, and $u_{0}$ is the small perturbation parameter. Substituting (2) into (1) will get the following equation:

$$
\frac{6 \gamma}{\beta}(\ln f)_{t t}+12 \gamma u_{0}(\ln f)_{x x}+\frac{36 \gamma^{2}}{\beta}\left((\ln f)_{x x}\right)^{2}+\frac{6 \gamma^{2}}{\beta}(\ln f)_{x x x x}=0
$$

By means of the hirota bilinear operator, which is defined by

$$
D_{x}^{m} D_{t}^{n} f(x, t) g(x, t)=\left(\frac{\partial}{\partial x}-\frac{\partial}{\partial x^{\prime}}\right)^{m} \times\left.\left(\frac{\partial}{\partial t}-\frac{\partial}{\partial t^{\prime}}\right)^{n} f(x, t) g\left(x^{\prime}, t^{\prime}\right)\right|_{x^{\prime}=x, t^{\prime}=t}
$$

we will get

$$
\begin{gathered}
\frac{\partial^{2}}{\partial x^{2}}(\ln f)=\frac{D_{x}^{2}(f \cdot f)}{2 f^{2}} \\
\frac{\partial^{4}}{\partial x^{4}}(\ln f)=\frac{D_{x}^{4}(f \cdot f)}{2 f^{2}}-\frac{3}{2}\left(\frac{D_{x}^{2}(f \cdot f)}{f^{2}}\right)^{2}
\end{gathered}
$$

Putting (5) (6) into (3) implies the following bilinear equation:

$$
\left(D_{t}^{2}+\beta u_{0} D_{x}^{2}+\gamma D_{x}^{4}\right)(f \cdot f)=0
$$

In this case we choose extended homoclinic test function 


$$
f=\mathrm{e}^{-p_{1}\left(x-w_{1} t\right)}+c_{1} \cos \left(p_{2}\left(x+w_{2} t\right)\right)+c_{2} \mathrm{e}^{p_{1}\left(x-w_{1} t\right)}
$$

where $p_{1}, p_{2}, w_{1}, w_{2}, c_{1}$ and $c_{2}$ are real constants to be determined.

Substituting Equation (8) into (7), collecting coefficients of the terms $\mathrm{e}^{p_{1}\left(x-w_{1} t\right)}, \mathrm{e}^{-p_{1}\left(x-w_{1} t\right)}, \sin \left(p_{2}\left(x+w_{2} t\right)\right)$, $\cos \left(p_{2}\left(x+w_{2} t\right)\right)$ and the constant, and let coefficients of these terms to zero, we get an algebraic equation

$$
\left\{\begin{array}{l}
2 c_{1} p_{1} p_{2} w_{1} w_{2}-4 \beta u_{0} c_{1} p_{1} p_{2}+4 \gamma c_{1} p_{1} p_{2}\left(-p_{1}^{2}+p_{2}^{2}\right)=0 \\
-2 c_{1} c_{2} p_{1} p_{2} w_{1} w_{2}+4 \beta u_{0} c_{1} c_{2} p_{1} p_{2}+4 \gamma c_{1} c_{2} p_{1} p_{2}\left(p_{1}^{2}-p_{2}^{2}\right)=0 \\
c_{1} c_{2}\left(p_{1}^{2} w_{1}^{2}-p_{2}^{2} w_{2}^{2}\right)+2 \beta u_{0} c_{1} c_{2}\left(p_{1}^{2}-p_{2}^{2}\right)+\gamma c_{1} c_{2}\left(p_{1}^{4}+p_{2}^{4}\right)-6 \gamma c_{1} c_{2} p_{1}^{2} p_{2}^{2}=0 \\
4 \gamma c_{1}^{2} p_{2}^{4}-2 \beta c_{1}^{2} p_{2}^{2} u_{0}+16 \gamma c_{2} p_{1}^{4}-c_{1}^{2} p_{2}^{2} w_{2}^{2}+8 \beta c_{2} p_{1}^{2} u_{0}+4 c_{2} p_{1}^{2} w_{1}^{2}=0 \\
\gamma c_{1} p_{1}^{4}-6 \gamma c_{1} p_{1}^{2} p_{2}^{2}+\gamma c_{1} p_{2}^{4}+2 \beta c_{1} p_{1}^{2} u_{0}-2 \beta c_{1} p_{2}^{2} u_{0}+c_{1} p_{1}^{2} w_{1}^{2}-c_{1} p_{2}^{2} w_{2}^{2}=0
\end{array}\right.
$$

Solving Equation (9), then taking $p_{2}=p_{1}$, we have

$$
c_{1}= \pm 2 \sqrt{\frac{\left(2 \gamma p_{1}^{2} w_{2}^{2}+2 \beta^{2} u_{0}^{2}+\beta u_{0} w_{2}^{2}\right) c_{2}}{-4 \gamma p_{1}^{2} w_{2}^{2}+2 \beta^{2} u_{0}^{2}+\beta u_{0} w_{2}^{2}}}, \quad w_{1} w_{2}=2 \beta u_{0}, \quad p_{1}^{2}=\frac{1}{4 \gamma}\left(w_{1}^{2}-w_{2}^{2}\right)
$$

where $w_{1}, w_{2}, c_{2}$ are some free real constants. Choosing $\beta u_{0} \neq 0$ and $c_{2}>0$, we get from(10) $\left|w_{1}\right|>\left|w_{2}\right|$.

Substituting (10) into (8), we get

$$
\begin{aligned}
& f_{1}(x, t)=2 \sqrt{c_{2}} \cosh \left(p_{1}\left(x-\frac{2 \beta u_{0}}{w_{2}} t\right)+\ln \sqrt{c_{2}}\right)+h_{1} \cos \left(p_{2}\left(x+w_{2} t\right)\right) \\
& \text { and } f_{2}(x, t)=2 \sqrt{c_{2}} \cosh \left(p_{1}\left(x-\frac{2 \beta u_{0}}{w_{2}} t\right)+\ln \sqrt{c_{2}}\right)-h_{1} \cos \left(p_{2}\left(x+w_{2} t\right)\right) .
\end{aligned}
$$

where $h_{1}=2 \sqrt{\frac{\left(2 \gamma p_{1}^{2} w_{2}^{2}+2 \beta^{2} u_{0}^{2}+\beta u_{0} w_{2}^{2}\right) c_{2}}{-4 \gamma p_{1}^{2} w_{2}^{2}+2 \beta^{2} u_{0}^{2}+\beta u_{0} w_{2}^{2}}}, \quad p_{1}= \pm \frac{1}{2} \sqrt{\frac{w_{1}^{2}-w_{2}^{2}}{\gamma}}, \quad w_{1}, w_{2} \in R$. Substituting (11) into

yields the solutions of (1) as follows, respectively

$$
\begin{aligned}
& u_{1}(x, t)=u_{0}+\frac{6 \gamma}{\beta} \frac{-4 h_{1} p_{1}^{2} 2 \sqrt{c_{2}} \sinh \left(p_{1}\left(x-\frac{2 \beta u_{0}}{w_{2}} t\right)+\ln \sqrt{c_{2}}\right) \sin \left(p_{2}\left(x+w_{2} t\right)\right)-h_{1}^{2} p_{1}^{2}+4 c_{2} p_{1}^{2}}{\left(2 \sqrt{c_{2}} \cosh \left(p_{1}\left(x-\frac{2 \beta u_{0}}{w_{2}} t\right)+\ln \sqrt{c_{2}}\right)+h_{1} \cos \left(p_{2}\left(x+w_{2} t\right)\right)\right)^{2}} \\
& u_{2}(x, t)=u_{0}+\frac{6 \gamma}{\beta} \frac{-4 h_{1} p_{1}^{2} 2 \sqrt{c_{2}} \sinh \left(p_{1}\left(x-\frac{2 \beta u_{0}}{w_{2}} t\right)+\ln \sqrt{c_{2}}\right) \sin \left(p_{2}\left(x+w_{2} t\right)\right)-h_{1}^{2} p_{1}^{2}+4 c_{2} p_{1}^{2}}{\left(2 \sqrt{c_{2}} \cosh \left(p_{1}\left(x-\frac{2 \beta u_{0}}{w_{2}} t\right)+\ln \sqrt{c_{2}}\right)-h_{1} \cos \left(p_{2}\left(x+w_{2} t\right)\right)\right)^{2}}
\end{aligned}
$$

The solution $u_{1}(x, t)$ (or $u_{2}(x, t)$ ) shows a new family of two-wave, breather solitary wave, which is a solitary wave and also is a periodic wave.

Substituting $c_{2}=1$ into the solution $u_{2}(x, t)$, it can be rewritten as follows

$$
u_{2}^{(1)}(x, t)=u_{0}+\frac{6 \gamma}{\beta} \frac{p_{1}^{2}\left(1-k_{1}^{2}-2 k_{1} \sinh \left(p_{1}\left(x-\frac{2 \beta u_{0}}{w_{2}} t\right)\right) \sin \left(p_{2}\left(x+w_{2} t\right)\right)\right)}{\left(\cosh \left(p_{1}\left(x-\frac{2 \beta u_{0}}{w_{2}} t\right)\right)-k_{1} \cos \left(p_{2}\left(x+w_{2} t\right)\right)\right)^{2}}
$$


where $k_{1}=\sqrt{\frac{2 \gamma p_{1}^{2} w_{2}^{2}+2 \beta^{2} u_{0}^{2}+\beta u_{0} w_{2}^{2}}{-4 \gamma p_{1}^{2} w_{2}^{2}+2 \beta^{2} u_{0}^{2}+\beta u_{0} w_{2}^{2}}}$ (see Figure 1).

Now we consider a limit behavior of $u_{2}^{(1)}(x, t)$ as the period $\frac{2 \pi}{p_{1}}$ of periodic wave $\cos \left(p_{1}\left(x+w_{2} t\right)\right)$ goes to infinite, i.e. $p_{1} \rightarrow 0$. By computing, we get the following result

$$
U_{\text {roguewave }}=u_{0}+\frac{24 \gamma\left(R-2\left(x-\frac{2 \beta u_{0}}{w_{2}} t\right)\left(x+w_{2} t\right)\right)}{\beta\left(\left(x-\frac{2 \beta u_{0}}{w_{2}} t\right)^{2}+\left(x+w_{2} t\right)^{2}+R\right)^{2}}
$$

where $R=\frac{-6 \gamma w_{2}^{2}}{2 \beta^{2} u_{0}^{2}+\beta u_{0} w_{2}^{2}}$, and $k_{1} \rightarrow 0$ and $w_{1}=w_{2}$ as $p_{1} \rightarrow 0$ (see Figure 2).

Especially, if let $u_{0}=0$, we will get $c_{2}<0$, so the two breather wave solution can not be obtained, meanwhile, the rational breather wave solution (rogue wave solution) can't also be find. The small perturbation parameter $u_{0}=0$ plays a huge part in finding rouge wave solution.

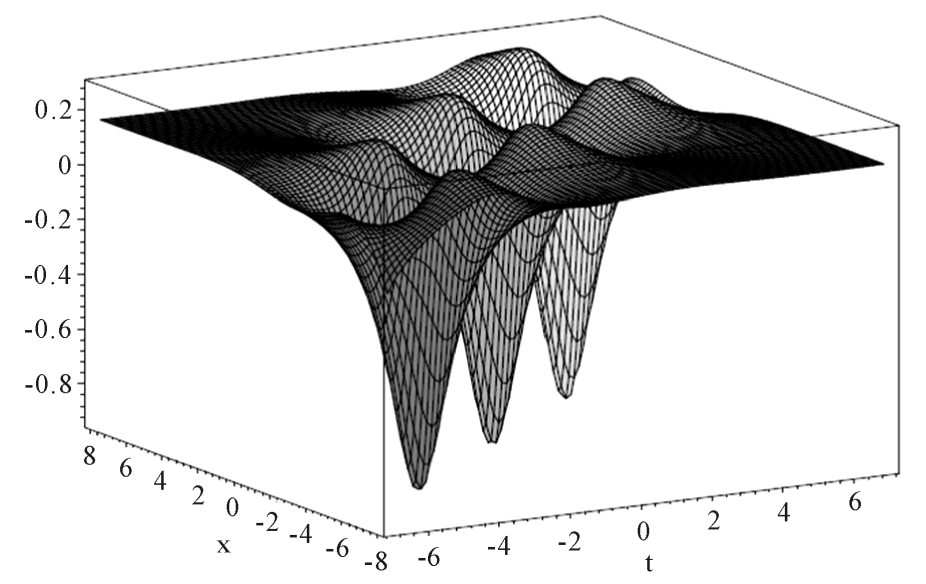

Figure 1. The figure of $u_{2}^{(1)}(x, t)$ as $u_{0}=\frac{1}{6}, \beta=6, \gamma=-1$.

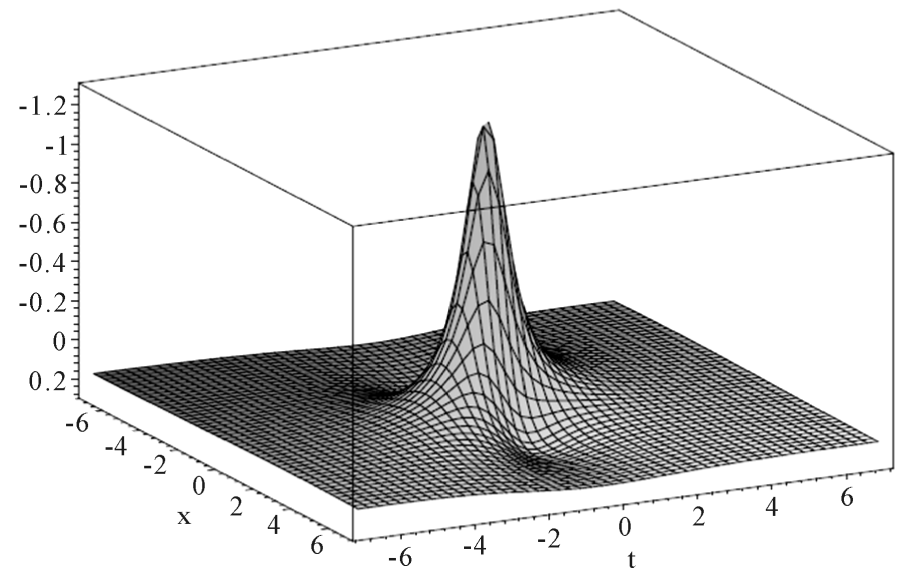

Figure 2. The figure of $U_{\text {roguewave }}$ as $u_{0}=\frac{1}{6}, \beta=6, \gamma=-1$. 
Equation (15) is a rational solution of Equation (1), and it is also a breather-type solution. $U \rightarrow 0$ for fixed $t$ as $x \rightarrow \pm \infty$. So, the solution $U_{\text {roguewave }}$ is a rogue wave solution which has two to three times amplitude higher than its surrounding waves and forms in a short time. One may think that whether the energy collection and superposition of breather solitary wave in many periods lead to a rogue wave or not.

\section{Conclusion}

In the paper, we apply the homoclinic (hateroclinic) breather limit method (HBLM) to find the BO equation's breather solitary solution and rational breather solution. Meanwhile, rational breather solution obtained here is just a rogue wave solution of the BO equation. Furthermore, the small perturbation parameter $u_{0}$ plays an important role in seeking rouge wave solution too. Next, we will try to use some methods to look for multi-rogue waves, such as the two-order wronskian determinant, Darboux transformation and so on.

\section{Acknowledgements}

The authors are grateful to the referee for a number of helpful suggestions to improve the paper.

\section{References}

[1] Ablowitz, M.J. and Clarkson, P.A. (1991) Solitons, Nonlinear Evolution Equations and Inverse Scattering. Cambridge University Press, Cambridge. http://dx.doi.org/10.1017/CBO9780511623998

[2] Korpel, A. and Banerjee, P. (1984) Heuristic Guide to Nonlinear Dispersive Wave Equation and Soliton-Type Solution. Proceedings of the IEEE, 72, 1109-1130. http://dx.doi.org/10.1109/PROC.1984.12992

[3] Fan, E. (2004) The Integrable Systems and The Computer Algebra. Science Press, Beijing.

[4] Fu. Z., Liu, S., et al. (2003) The JEFE Method and Periodic Solutions of Two Kinds of Nonlinear Wave Equations. Communications in Nonlinear Science and Numerical Simulation, 8, 67-75. http://dx.doi.org/10.1016/S1007-5704(02)00082-5

[5] Wang, Z., Li, D., et al. (2005) A Method for Constructing Exact Solutions and Application to Benjamin Ono Equation. Chinese Physics, 14, 2158-2163. http://dx.doi.org/10.1088/1009-1963/14/11/003

[6] Dai, Z., Huang, J., et al. (2005) Homoclinic Orbits and Periodic Solitons for Boussinesq Equation with Even Constraint. Chaos, Solitons \& Fractals, 26, 1189-1194. http://dx.doi.org/10.1016/j.chaos.2005.02.025

[7] Dai, Z., Jiang, M., et al. (2006) Homoclinic Bifurcation for Boussinesq Equation with Even Constraint. Chinese Physics Letters, 23, 1065-1067. http://dx.doi.org/10.1088/0256-307X/23/5/001

[8] Dai, Z., Liu, J. and Li, D. (2009) Applications of HTA and EHTA to YTSF Equation. Applied Mathematics and Computation, 207, 360-364. http://dx.doi.org/10.1016/j.amc.2008.10.042

[9] Dai, Z., Li, Z., et al. (2008) Exact Homoclinic Wave and Soliton Solutions for the 2D Ginzburg-Landau Equation. Physics Letters A, 372, 3010-3014. http://dx.doi.org/10.1016/j.physleta.2008.01.015

[10] Dai, Z., Liu, Z. and Li, D. (2008) Exact Periodic Solitary-Wave Solution for KdV Equation. Chinese Physics Letters, 25, 1531-1533. http://dx.doi.org/10.1088/0256-307X/25/5/003

[11] Xu, Z.H., Xian, D.Q. and Chen, H.L. (2010) New Periodic Solitary-Wave Solutions for the Benjamin Ono Equation. Applied Mathematics and Computation, 215, 4439-4442. http://dx.doi.org/10.1016/j.amc.2009.11.009

[12] Ohta, Y. and Yang, J.K. (2012) General High-Order Rogue Waves and Their Dynamics in the Nonlinear Schrödinger Equation. Proceedings of the Royal Society A: Mathematical, Physical and Engineering Sciences, 468, 1716-1740.

[13] Muller, P., Garrett, C. and Osborne, A. (2005) Rouge Waves. Oceanography, 18, 66-75.

[14] Kharif, C., Pelinovsky, E. and Slunyaey, A. (2009) Rogue Waves in the Ocean, Observation, Theories and Modeling. Springer, New York.

[15] Akhmediev, N., Ankiewicz, A. and Soto-Crespo, J.M. (2009) Rogue Waves and Rational Solutions of the Nonlinear Schrödinger Equation. Physical Review E, 80, Article ID: 026601. http://dx.doi.org/10.1103/PhysRevE.80.026601

[16] Solli, D.R., Ropers, C., Koonath, P. and Jalali, B. (2007) Optical Rogue Waves. Nature, 450, 1054-1057. http://dx.doi.org/10.1038/nature06402

[17] Bludov, V.Y., Konotop, V.V. and Akhmediev, N. (2009) Rogue Waves as Spatial Energy Concentrators in Arrays of Nonlinear Waveguides. Optics Letters, 34, 3015-3017. http://dx.doi.org/10.1364/OL.34.003015

[18] Ganshin, A.N., Efimov, V.B., Kolmakov, G.V., Mezhov-Deglin, L.P. and McClintock, P.V.E. (2008) Statistical Properties of Strongly Nonlinear Waves within a Resonator. Physical Review Letters, 101, Article ID: 065303. 
http://dx.doi.org/10.1103/PhysRevLett.101.065303

[19] Bludov, V.Y., Konotop, V.V. and Akhmediev, N. (2009) Matter Rogue Waves. Physical Review A, 80, Article ID: 033610. http://dx.doi.org/10.1103/PhysRevA.80.033610

[20] Montina, A., Bortolozzo, U., Residori, S. and Arecchi, F.T. (2013) Rogue Waves and Their Generating Mechanisms in Different Physical Contexts. Physics Reports, 528, 47-89. http://dx.doi.org/10.1016/j.physrep.2013.03.001

[21] Solli, D.R., Ropers, C. and Jalali, B. (2008) Active Control of Optical Rogue Waves for Stimulated Supercontinuum Generation. Physical Review Letters, 101, Article ID: 233902. http://dx.doi.org/10.1103/PhysRevLett.101.233902

[22] Yan, Z.Y. (2011) Vector Financial Rogue Waves. Physics Letters A, 375, 4274-4279. http://dx.doi.org/10.1016/j.physleta.2011.09.026

[23] Xu, Z.H., Chen, H.L. and Dai, Z.D. (2014) Rogue Wave for the (2+1)-Dimensional Kadomtsev-Petviashvili Equation. Applied Mathematics Letters, 37, 34-38. http://dx.doi.org/10.1016/j.aml.2014.05.005

[24] Hirota, R. (1985) Fundamental Properties of the Binary Operators in Soliton Theory and Their Generalizartion. In: Takeno, S., Ed., Dynamical Problem in Soliton Systems, Springer Series in Synergetiecs, Springer, Berlin, 42-49.

[25] Dai, Z.D., Liu, J. and Li, D.L. (2009) Applications of HTA and EHTA to YTSF Equation. Applied Mathematics and Computation, 207, 360-364. http://dx.doi.org/10.1016/j.amc.2008.10.042 
Scientific Research Publishing (SCIRP) is one of the largest Open Access journal publishers. It is currently publishing more than 200 open access, online, peer-reviewed journals covering a wide range of academic disciplines. SCIRP serves the worldwide academic communities and contributes to the progress and application of science with its publication.

Other selected journals from SCIRP are listed as below. Submit your manuscript to us via either submit@scirp.org or Online Submission Portal.
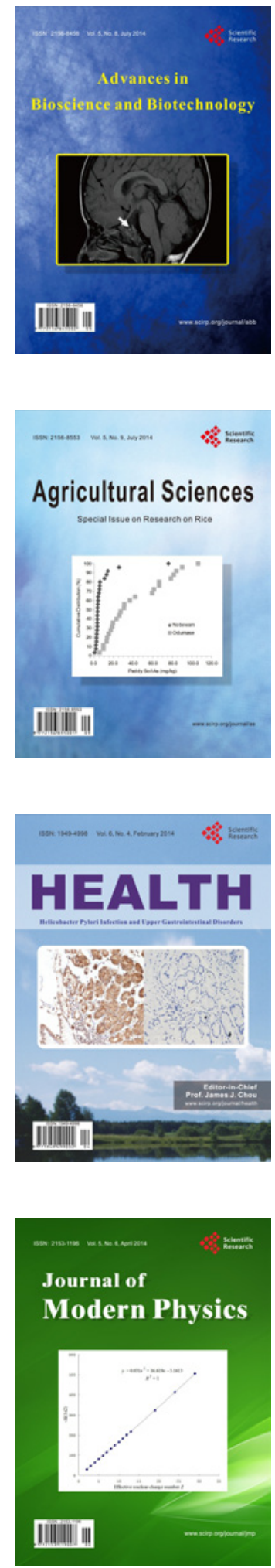
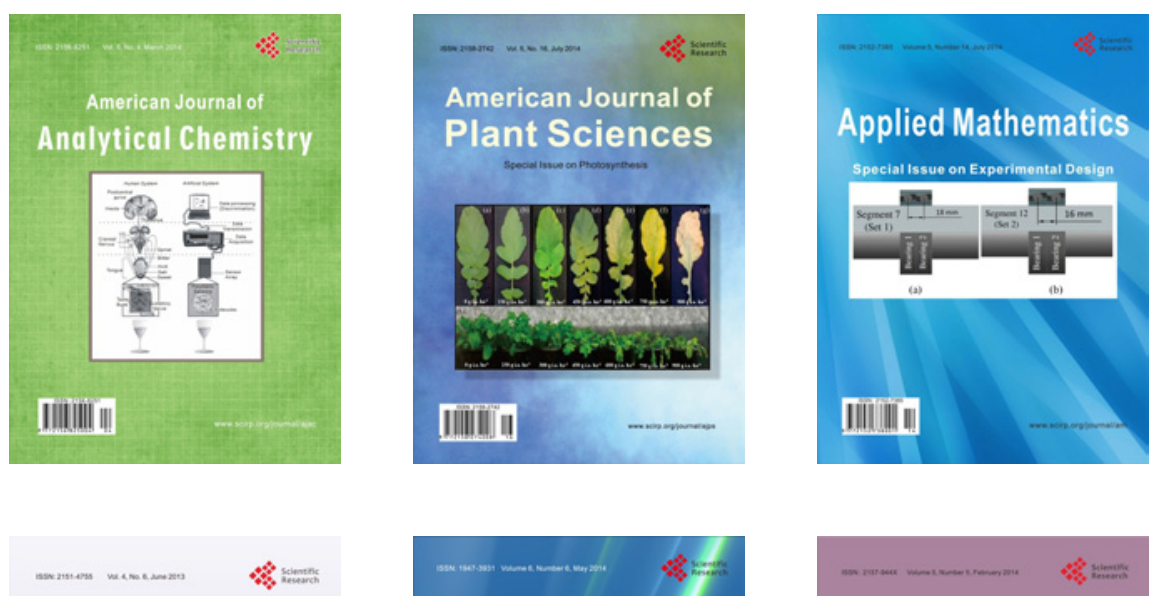

Creative Education
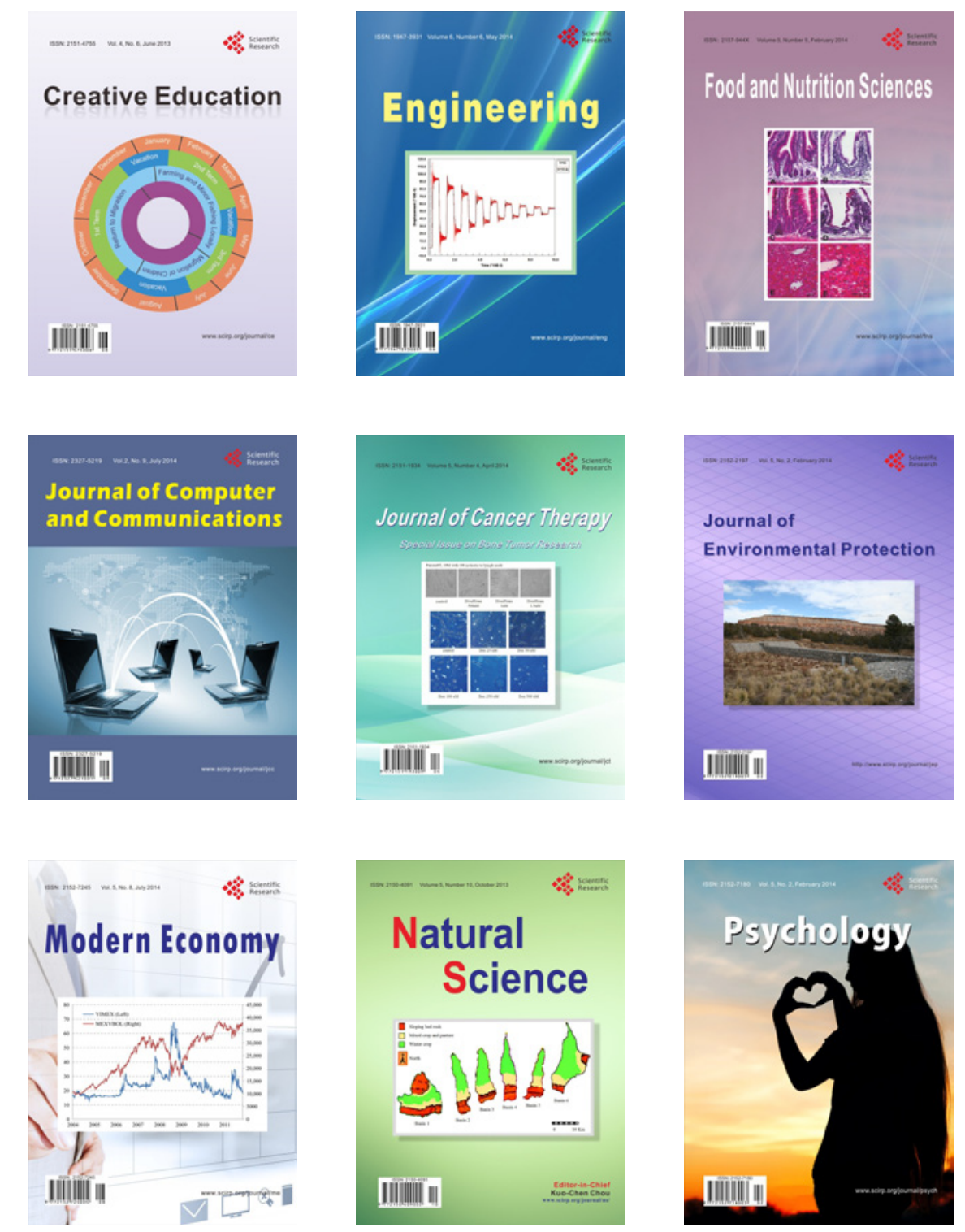